This is an electronic reprint of the original article. This reprint may differ from the original in pagination and typographic detail.

Author(s): Marinov, Corneliu A.; Neittaanmäki, Pekka

Title: $\quad$ A Theory of electrical circuits with resistively coupled distributed structures : Delay time predicting

Year: $\quad 1988$

Version:

Please cite the original version:

Marinov, C. A.\& Neittaanmäki, P. (1988). A Theory of electrical circuits with resistively coupled distributed structures : Delay time predicting. IEEE Transactions on Circuits and Systems , 35 (2), 173-183.

All material supplied via JYX is protected by copyright and other intellectual property rights, and duplication or sale of all or part of any of the repository collections is not permitted, except that material may be duplicated by you for your research use or educational purposes in electronic or print form. You must obtain permission for any other use. Electronic or print copies may not be offered, whether for sale or otherwise to anyone who is not an authorised user. 


\title{
A Theory of Electrical Circuits with Resistively Coupled Distributed Structures: Delay Time Predicting
}

\author{
CORNELIU A. MARINOV AND PEKKA NEITTAANMÄKI
}

\begin{abstract}
This paper deals with qualitative properties (existence, uniqueness, and especially, stability) and numerical solution of a circuit consisting of a resistive multiport with $\boldsymbol{r}-\boldsymbol{c}$-g "exactly modeled" distributed elements connected to its terminals. This kind of results are useful, for instance, when we study the effect of interconnections on the speed of transient process from an integrated structure. A formula to evaluate the delay time as a global parameter of the circuit is given and verified by numerical calculus.
\end{abstract}

\section{INTRODUCTION}

$\mathrm{T}$ HE technology of integrated circuits is structurally involved in dealing with structures with distributed parameters. For instance, in MOS integrated circuits, a given inverter or logic node may drive several gates through polysilicon thin-film wires. This thin-film technology results in interconnection lines with parameters distributed over their entire length. Also, in monolithic structures a resistor or a capacitor appears unavoidably as a component presenting as well a distributed resistance $(r)$, a distributed capacitance $(c)$, and a distributed conductance $(g)$. With the advances in technology, the delay time associated with interconnections becomes significant compared to the device delay time and in certain cases dominates the chip performance [1]. To predict this extradevices delay for an integrated structure, we can replace the devices with their resistive models and thus we have to study a network composed by a resistive multiport with $r-c-g$ distributed structures ("lines") connected to its terminals (see Fig. 1).

The mathematical model of this class of circuits (formulated in Section II) consists of a parabolic system of equations with nonstandard boundary conditions. The correctness of this model is given by existence, uniqueness and stability theorems enounced in Section III where the restrictions of hypotheses are also commented.

Our theorem regarding exponential asymptotic stability (the main result of this paper) suggests a definition and

\footnotetext{
Manuscript received October 16,1985; revised February 12, 1987. This paper was recommended by Associate Editor M.N.S. Swamy.

C. A. Marinov is with the Faculty of Electrotechnics, Polytechnical Institute of Bucharest, Bucharest 77206, Romania.

P. Neittaanmäki is with the Department of Mathematics, University of Jyväskylä, SF-40100 Jyväskylä, Finland.

IEEE Log Number 8718324.
}

allows an evaluation of this delay time of the circuit under study. This parameter characterizes the rate of the evolution of the state vector to the stationary regime. As the upper bound of this characteristic depends upon all the circuit parameters and is easy to compute, such an approach seems to be quite useful in circuit design.

The proofs (presented in Section IV) use essentially the dissipative operator approach (see [3], [4]) and its relations to abstract differential equation arising from our model.

In Section $\mathrm{V}$ a numerical algorithm of the type finite elements in space, finite differences in time is formulated in order to obtain the solution of the problem under study. This algorithm is then used in Section VI in validating the evaluation of the response time computed for several examples.

The main mathematical notations and definitions are relegated to Appendix where a useful lemma is also proved.

If we try to establish the relations of this paper with mathematical and technical literature, let us mention at the beginning that the nonclassical kind of boundary conditions appearing in our model were considered in [5]-[8] but for hyperbolic problems which cannot come to our one.

The stability of the circuits with distributed structures was studied by using different methods. In the remarkable paper [9], for instance, the authors use a Lyapunov theory for a functional-differential equation of retarded type that describe a large class of such circuits. A small signal stability criterion (in terms of the roots of characteristic equation) is derived in [10] and input-output stability results are given in [11] for circuits with parasitic elements, by applying the theory of singular perturbations.

Analytical estimations of the transient response of circuits containing distributed structures ( $r-c$ lines) were obtained especially for the case of a single such distributed element. A pioneering work in this field is [12]. For a nonuniform open circuited $r$-c line the paper [13] shows the step response to be monotonously increasing. In [14] the bounds of this response were obtained.

The engineering literature shows lately an intense preoccupation regarding the estimation of signals delay in MOS integrated circuits (see [2], [15]-[19] and the references 


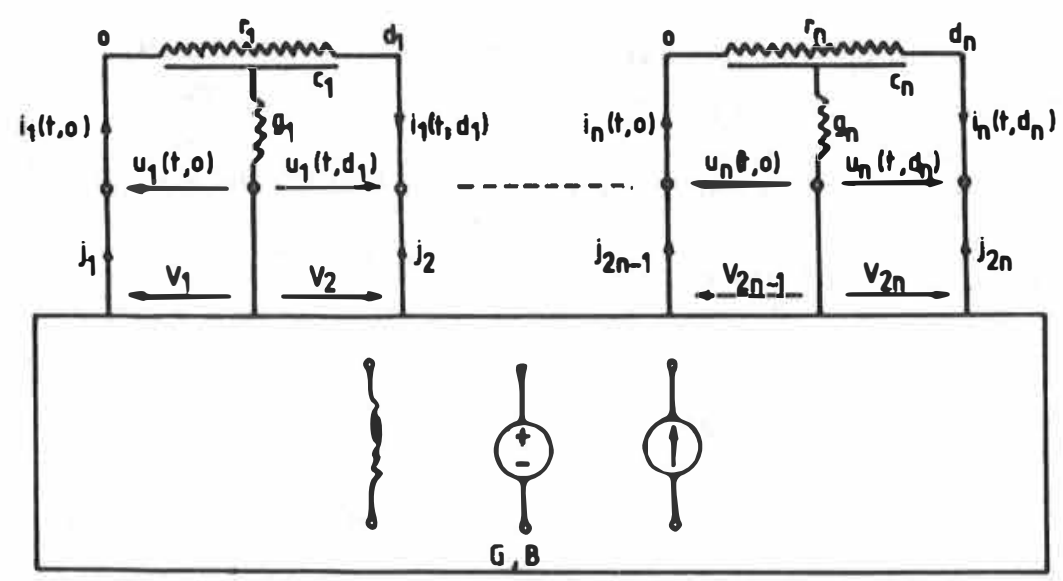

Fig. 1. The circuit under study.

quoted therein); the approximation of distributed elements by $R C$ ladder networks (even infinite $R C$ networks [20]) is used throughout. The accuracy of such an approximation is rarely studied and then for a single distributed element [15].

The delay estimation in lumped parameter $R C$ circuits allows efficient using of CAD programs ([1], [17], [18] and references quoted therein). A fundamental paper in this respect is [2], where bounds of the transient response at a step-variation input are derived for each node of a lumped $R C$ fanout circuit. The methods and results of this paper were then extended to mesh-type networks, [18], and to $R C$ ladder networks with nonlinearities, [19]. Another extension which takes into account the effect of initial condition is presented in [17].

There are two main directions of study that arise naturally; first, to consider circuits whose distributed elements are "exact" models ( $r$ - $c$-g lines) and, second, to consider the dynamic behavior excited by step-variations simultaneously applied to more than one input. With such significant extensions of the model, the present paper gives the estimation of the delay time defined as a global parameter of the circuit.

We shall report elsewhere the extension of below results to the circuits supplementary containing lumped capacitors. A preliminary restricted version of this paper were [21], [30].

\section{The Circuit Model}

The circuit under study consists of a lumped parameter resistive multiport with any kind of sources as well as $r-c-g$ distributed elements connected to its terminals, as shown in Fig. 1.

In Fig. $1, u_{k}(t, x)$ and $i_{k}(t, x)$ is, respectively, the voltage and the current at the moment $t \in[0, \infty)$ in the point $x \in\left[0, d_{k}\right]$ of the distributed structure, $k \in \overline{1, Q_{n}}$. This structure is mathematically described by the wellknown telegraph equations:

$$
\left\{\begin{array}{l}
\frac{\partial u_{k}(t, x)}{\partial x}=-r_{k} i_{k}(t, x) \\
\frac{\partial i_{k}(t, x)}{\partial x}=-c_{k} \frac{\partial u_{k}(t, x)}{\partial t}-g_{k} u_{k}(t, x) .
\end{array}\right.
$$

In the following we shall consider $r_{k}, c_{k}$, and $g_{k}$ constant parameters and we assume $r_{k}, c_{k}>0, g_{k} \geqslant 0$.

From (2.1) we formally derive the system:

$$
\begin{array}{r}
\frac{\partial u_{k}(t, x)}{\partial t}=\frac{1}{r_{k} c_{k}} \frac{\partial^{2} u_{k}(t, x)}{\partial x^{2}}-\frac{g_{k}}{c_{k}} u_{k}(t, x), \\
\quad x \in\left[0, d_{k}\right], t \geqslant 0, k \in \overline{1, n} .
\end{array}
$$

On the other hand, we shall suppose that the multiport ( $2 n$-port) is described by the following linear relation between the vector of the currents and the vector of voltages:

$$
\left[\begin{array}{c}
j_{1} \\
\vdots \\
j_{2 n}
\end{array}\right]=-G\left[\begin{array}{c}
v_{1} \\
\vdots \\
v_{2 n}
\end{array}\right]+B(t)
$$

where $G$ is a $2 n \times 2 n$ matrix of conductances and $B$ is a $2 n$ vector depending on sources. By observing in Fig. 1 that $i_{k}(t, 0)=j_{2 k-1}(t)$ and $i_{k}\left(t, d_{k}\right)=-j_{2 k}(t)$ and that $u_{k}(t, 0)=v_{2 k-1}(t)$ and $u_{k}\left(t, d_{k}\right)=v_{2 k}(t)$ we can derive from the first relation (2.1) and from (2.3) the following system of boundary conditions:

$$
\left[\begin{array}{c}
-\frac{1}{r_{1}} \frac{\partial u_{1}}{\partial x}(t, 0) \\
\frac{1}{r_{1}} \frac{\partial u_{1}}{\partial x}\left(t, d_{1}\right) \\
\vdots \\
-\frac{1}{r_{n}} \frac{\partial u_{n}}{\partial x}(t, 0) \\
\frac{1}{r_{n}} \frac{\partial u_{n}}{\partial x}\left(t, d_{n}\right)
\end{array}\right]=-G\left[\begin{array}{c}
u_{1}(t, 0) \\
u_{1}\left(t, d_{1}\right) \\
\vdots \\
u_{n}(t, 0) \\
u_{n}\left(t, d_{n}\right)
\end{array}\right]+B(t) .
$$

Of course we have to add the initial condition:

$$
u_{k}(0, x)=u_{k 0}(x), \quad x \in\left[0, d_{k}\right], \quad k \in \overline{1, n}
$$

and let $u_{0}(x)$ the vector with $u_{k 0}(x)$ as components.

With these, our problem is the system (2.2) with boundary conditions (2.4) and with initial conditions (2.5). 


\section{Statement of the Results and Comments}

In this section we refer to the solutions in the classical sense of our problem. The first result deals with the sufficient conditions for existence and uniqueness of such a solution.

Theorem 3.1: Let us consider all independent sources having simultaneous step variation at $t=0$. Then

i) If $G$ is a positive semidefinite matrix and $u_{0}$ is a $C^{2}$ function satisfying the boundary conditions, the dynamic problem (2.2), (2.4), (2.5) has a unique solution.

ii) If $G$ is positive semidefinite and $g_{i}>0$ for all $i$ $\in \overline{1, n}$, the steady-state solution of our problem exists and is unique.

iii) If $G$ is positive definite and there is $i \in \overline{1, n}$ for which $g_{i}=0$, there exists a unique steady-state solution.

Our second result establishes sufficient conditions for global exponential asymptotic stability of the network solutions. Before enouncing it, let us observe that if $G$ is DRD we can find a unique $\gamma_{j} \in(0, \pi / 2)$ such that

$$
\cos \gamma_{j}=\frac{S_{2 j}+\sqrt{S_{2 j}^{2}+\frac{4}{r_{j} d_{j}}\left(G_{2 j, 2 j}+\frac{1}{r_{j} d_{j}}\right)}}{2\left(G_{2 j, 2 j}+\frac{1}{r_{j} d_{j}}\right)}
$$

for each $j \in \overline{1, n}$. For our $2 n \times 2 n$ matrix $G$ with $G_{i j}$ elements we shall denote

$$
S_{i}=\sum_{j=1, j \neq i}^{2 n}\left|G_{i j}\right|
$$

and we shall say that $G$ is diagonally row sum dominant (DRD) if for each $i$ we have $G_{i i}>S_{i}$. If $G_{i i} \geqslant S_{i}, G$ is said to be weakly diagonally row sum dominant (WDRD).

Also, if we denote

$$
\omega_{0}=-\min _{1 \leqslant j \leqslant n}\left(\frac{\gamma_{j}^{2}}{r_{j} c_{j} d_{j}^{2}}+\frac{g_{j}}{c_{j}}\right)
$$

we have:

Theorem 3.2: Let $G$ be a DRD matrix and $u, \bar{u}$ be two solutions of the problem (2.2), (2.4), (2.5) for two different $C^{2}$ initial conditions $u_{0}$ and $\bar{u}_{0}$, respectively, and for the same continuous sources. Then,

$$
\frac{\max _{1 \leqslant i \leqslant n} \max _{0 \leqslant x_{i} \leqslant d_{i}}\left|u_{i}\left(t, x_{i}\right)-\bar{u}_{i}\left(t, x_{i}\right)\right|}{\max _{1 \leqslant i \leqslant n} \max _{0 \leqslant x_{i} \leqslant d_{i}}\left|u_{i 0}\left(x_{i}\right)-\bar{u}_{i 0}\left(x_{i}\right)\right|} \leqslant \frac{e^{\omega_{0} t}}{\min _{1 \leqslant i \leqslant n} \cos \gamma_{i}} .
$$

Moreover, the time function from the left-hand side of above inequality is strictly decreasing on $[0, \infty)$.

Now let us suppose that $u_{i}$ are the components of the solution for step sources, $u_{i 0}$ are their initial values and $\tilde{u}_{i} \neq u_{i 0}$ are the components of the solution of the time independent problem with the same step sources. The above theorem gives $\lim _{t \rightarrow \infty} u_{i}(t, x)=\tilde{u}_{i}(x)$ for every $i$ $\in \overline{1, n}$ and $x \in\left[0, d_{i}\right]$. The evolution of all $u_{i}$ functions (and, therefore, the evolution of the state of our network) from the initial state $u_{i 0}$ towards the steady state $\tilde{u}_{i}$ can be globally described by the function $D:[0, \infty) \rightarrow(0,1]$ with

$$
D(t)=\frac{\max _{1 \leqslant i \leqslant n} \max _{0 \leqslant x_{i} \leqslant d_{i}}\left|u_{i}\left(t, x_{i}\right)-\tilde{u}_{i}\left(x_{i}\right)\right|}{\max _{1 \leqslant i \leqslant n} \max _{0 \leqslant x_{i} \leqslant d_{i}}\left|u_{i 0}\left(x_{i}\right)-\tilde{u}_{i}\left(x_{i}\right)\right|} .
$$

In the assumptions of Theorem 3.2 it holds

$$
D(t) \leqslant \bar{D}(t)=\frac{e^{\omega_{0} t}}{\min _{1 \leqslant i \leqslant n} \cos \gamma_{i}} .
$$

If we fix $\lambda \in(0,1)$ let us define the " $\lambda$ delay time" of the circuit as the last instant when $D(t)$ passes through $\lambda$ value, i.e.,

$$
T_{\lambda}=\sup _{t \geqslant 0}\{t ; D(t)=\lambda\} .
$$

Theorem 3.2 allows us to write $T_{\lambda}=D^{-1}(\lambda)$ and, more important, provides us an upper bound $\bar{T}_{\lambda}$ of this parameter, namely,

$$
T_{\lambda} \leqslant \bar{T}_{\lambda}=\frac{\ln \lambda \min _{1 \leqslant i \leqslant n} \cos \gamma_{i}}{\omega_{0}} .
$$

It is clear from its definition that $T_{\lambda}$ is a measure for the speed of signals propagation in our network. As its upper bound $\bar{T}_{\lambda}$ can be easily computed from circuit parameters (see (3.1), (3.2), (3.6)) and is sufficiently tight (see examples in Section VI), it can be useful for initial design stages.

In order to apply the previous results for circuit models validation, one has to check the assumptions used in obtaining those results. Consider the usual case of the MOS interconnections analysis [2], when:

a) one of the multiport terminals is common for all external elements (this is the common "ground" of the $r-c-g$ lines)

b) all sources are independent.

A straightforward analysis of the resistive multiports having the properties a) and b), [22], shows that:

1) The existence of the matrix $G$ is ensured if and only if the following hold:

c) none of the 0 or $d_{i}$-terminals is connected directly (i.e., through a zero-resistance branch) to the ground

d) there is no direct connection between 0 and/or $d_{i}$ terminals.

2) If $G$ does exist, then it is symmetric and WDRD, hence it is positively semidefinite.

All of above facts imply that if, besides the assumptions a)-d), is required that $g_{i}>0$ for all $i$ then, according to Theorem 3.1, we obtain a broad class of circuits-let us 


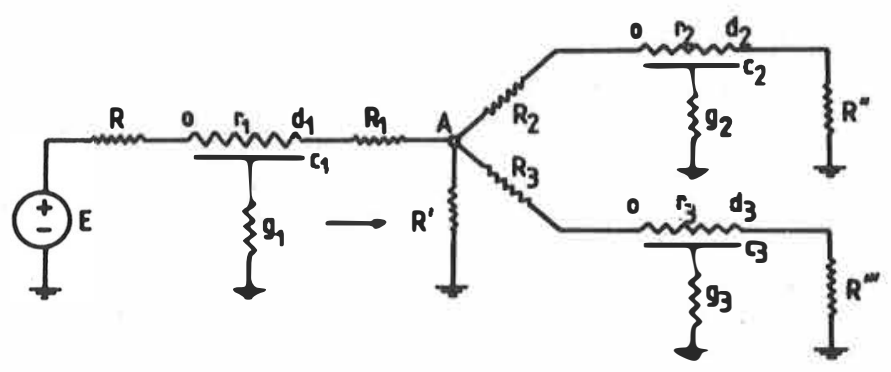

Fig. 2. The simplest fanout circuit.

say $\mathscr{C}$ - for which the dynamic and dc solutions exist and are unique.

In order to ensure the realization of the assumptions of Theorem 3.2 (thus allowing the delay time estimation) one has to add the DRD condition for $G$ to conditions a)-d). Due to the fact that in this case $G$ is positive definite, existence and uniqueness of the solution hold even if $g_{i}=0$ for some $i$.

Consider, for instance, the simplest interconnection circuit of Fig. 2 where the three distributed elements are connected in A and the circuit loads are $R^{\prime \prime}, R^{\prime \prime \prime} \in(0, \infty)$.

If, as usually, we take $R_{1}=R_{2}=R_{3}=0$ and $R^{\prime}=\infty$, the condition c) is not fulfilled hence the matrix $G$ does not exist. However if we assume that $R^{\prime}=\infty$ but $R_{1}, R_{2}, R_{3} \neq$ 0 (the contact resistances) and $g_{1}, g_{2}, g_{3}>0$, the circuit belongs to class $\mathscr{C}$, hence its model is correct. Unfortunately, one can still say nothing about the stability of the solutions.

Finally, if $R_{1}, R_{2}, R_{3} \neq 0$ and $R^{\prime}, R^{\prime \prime}, R^{\prime \prime \prime} \in(0, \infty)$, we find

\section{Proofs}

\section{A. Proof of Theorem 3.1}

We consider for the beginning the stationary (i.e., time independent) problem, where we take zero-dielectric conductivity for the first $k_{0}$ distributed elements. So we have

$$
\begin{aligned}
& \frac{\partial^{2} \tilde{u}_{k}}{\partial x^{2}}-a_{k} \tilde{u}_{k}=0, \quad k \in \overline{1, n} \\
& {\left[\begin{array}{c}
-\frac{1}{r_{1}} \frac{\partial \tilde{u}_{1}}{\partial x}(0) \\
\frac{1}{r_{1}} \frac{\partial \tilde{u}_{1}}{\partial x}\left(d_{1}\right) \\
\vdots \\
-\frac{1}{r_{n}} \frac{\partial \tilde{u}_{n}}{\partial x}(0) \\
\frac{1}{r_{n}} \frac{\partial \tilde{u}_{n}}{\partial x}\left(d_{n}\right)
\end{array}\right]=-G\left[\begin{array}{c}
u_{1}(0) \\
u_{1}\left(d_{1}\right) \\
\vdots \\
u_{n}(0) \\
u_{n}\left(d_{n}\right)
\end{array}\right]+B}
\end{aligned}
$$

where $G_{i}=1 / R_{i}, i=1,2,3, G^{\prime}=1 / R^{\prime}$, and $M=G_{1}+G_{2}+$ $G_{3}+G^{\prime}$.

This matrix is DRD and then all the qualitative properties are valid regardless of $g_{i}$ values. A finite $R^{\prime}$ can be "created" by concentrating the dielectric conductance $g_{1}$ to the point $A$, i.e., $R^{\prime}=1 / g_{1} d_{1}$. The distributed element is taken into account afterwards via the nonzero parameters $r_{1}, c_{1}, d_{1}$, and $g_{1}=0$. The same approach can be taken for getting finite values for $R^{\prime \prime}$ and $R^{\prime \prime \prime}$ if the last two lines are originally open circuited. where we have denoted $a_{k}=g_{k} / r_{k}$ with $a_{k}=0$ for $k$

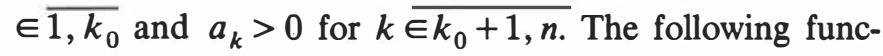
tions

$$
\begin{cases}\tilde{u}_{k}(x)=A_{k} x+B_{k}, & k \in \overline{1, k_{0}} \\ \tilde{u}_{k}(x)=A_{k} e^{-\sqrt{a_{k}} x}+B_{k} e^{-\sqrt{a_{k}} x}, & k \in \overline{k_{0}+1, n}\end{cases}
$$

verify (4.1). 
By checking the boundary conditions (4.2) for the functions (4.3) and using the notations $w_{2 k-1}=B_{k}$ and $w_{2 k}=$ $A_{k} d_{k}+B_{k}$ for $k \in \overline{1, k_{0}}$ and also $w_{2 k-1}=A_{k}+B_{k}$ and


linear equation in $\boldsymbol{R}^{2 n}$ of the form

$$
P w=-G w+B
$$

where $w$ is the $2 n$ vector with components $w_{k}$. The following estimation is easy to be derived for the matrix $P$ :

$$
\begin{aligned}
w^{T} P w \geqslant M_{1} \sum_{k=1}^{k_{0}}\left(w_{2 k-1}-w_{2 k}\right)^{2} & \\
& +M_{2} \sum_{k=k_{0}+1}^{n}\left(w_{2 k-1}^{2}+w_{2 k}^{2}\right)
\end{aligned}
$$

where $M_{1}$ and $M_{2}$ are strictly positive constants. Hence, the matrix $P+G$ is positive definite under each of our hypotheses ii) or iii), so that (4.4) has a solution which fixes the constants in (4.3).

The uniqueness of steady-state solution results from the dissipativity properties that follow. Let us define the operator $A: \mathscr{D}(A) \rightarrow H_{c}^{0}$ with

$$
\mathscr{D}(A)=\left\{u=\left(u_{1}, \cdots, u_{n}\right) \in H_{c}^{2}\right. \text { and satisfies }
$$

and

$$
A u=\left[\begin{array}{cc}
\frac{1}{r_{1} c_{1}} & \frac{\partial^{2} u_{1}}{\partial x^{2}}-\frac{g_{1}}{c_{1}} u_{1} \\
\cdots & \frac{1}{r_{n} c_{n}} \frac{\partial^{2} u_{n}}{\partial x^{2}}-\frac{g_{n}}{c_{n}} u_{n}
\end{array}\right], \quad \text { where } u \in \mathscr{D}(A) \subset H_{c}^{0} .
$$

If we take $\bar{u}, \overline{\bar{u}} \in \mathscr{D}(A)$, then an elementary calculation shows, that

$$
\begin{aligned}
\langle\bar{u}-\overline{\bar{u}}, A \bar{u}-A \overline{\bar{u}}\rangle_{H_{c}^{0}} & \\
\leqslant & \sum_{k=1}^{n}\left(-\frac{1}{r_{k}} \frac{\partial u_{k}}{\partial x}(0) \cdot u_{k}(0)+\frac{1}{r_{k}} \frac{\partial u_{k}}{\partial x}\left(d_{k}\right) \cdot u_{k}\left(d_{k}\right)\right) \\
= & -\left[\left(u_{1}(0), u_{1}\left(d_{1}\right), \cdots, u_{n}\left(d_{n}\right)\right)\right. \\
& \left.\cdot G\left(u_{1}(0), u_{1}\left(d_{1}\right), \cdots, u_{n}\left(d_{n}\right)\right)\right]^{T}
\end{aligned}
$$

where $u_{k}$ are the components of vector $u=\bar{u}-\overline{\bar{u}}$. When $G$ is positive semidefinite (4.5) implies that $A$ is dissipative. The notation $H_{c_{k}}^{l}\left(0, d_{k}\right), l \geqslant 0$, is used for the usual Sobolev space (with a weight $c_{k}$ ) of real functions defined on $\left(0, d_{k}\right)$. Particularly $L_{c_{k}}^{2}\left(0, d_{k}\right)=H_{c_{k}}^{0}\left(0, d_{k}\right)$. Consider also $H_{c}^{l}=\prod_{k=1}^{n} H_{c_{k}}^{l}\left(0, d_{k}\right)$ and $H^{l}=\prod_{k=1}^{n} H_{1}^{l}\left(0, d_{k}\right)$. The inner product in $H_{c}^{0}$ will be $\langle u, v\rangle_{H_{c}^{0}}=\sum_{k=1}^{n} c_{k} \int_{0}^{d_{k}} u(x)$ $v(x) d x$.

In order to prove the $m$-dissipativity we have to find a function $u \in \mathscr{D}(A)$ such as for each component

$$
\frac{\partial^{2} u_{k}}{\partial x^{2}}-a_{k} u_{k}=v_{k}
$$

where $v_{k} \in H_{c_{k}}^{0}\left(0, d_{k}\right)$ and $a_{k}>0$. This problem splits into two simpler problems:

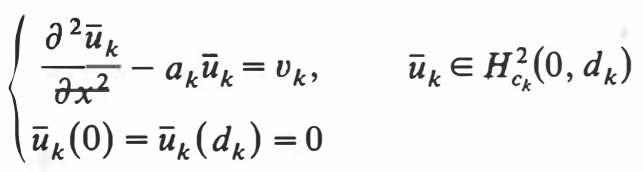

and

$\left\{\begin{array}{c}\frac{\partial^{2} \tilde{u}_{k}}{\partial x^{2}}-a_{k} \tilde{u}_{k}=0, \quad \tilde{u}_{k} \in H_{c_{k}}^{2}\left(0, d_{k}\right) \\ {\left[\begin{array}{c}-\frac{1}{r_{1}} \frac{\partial \tilde{u}_{1}}{\partial x}(0) \\ \frac{1}{r_{1}} \frac{\partial \tilde{u}_{1}}{\partial x}\left(d_{1}\right) \\ \vdots \\ \frac{1}{r_{n}} \frac{\partial \tilde{u}_{n}}{\partial x}\left(d_{n}\right)\end{array}\right]=-G\left[\begin{array}{c}\tilde{u}_{1}(0) \\ \tilde{u}_{1}\left(d_{1}\right) \\ \vdots \\ \tilde{u}_{n}(0) \\ \tilde{u}_{n}\left(d_{n}\right)\end{array}\right]-\left[\begin{array}{c}-\frac{1}{r_{1}} \frac{\partial \bar{u}_{1}}{\partial x}(0) \\ \frac{1}{r_{1}} \frac{\partial \bar{u}_{1}}{\partial x}\left(d_{1}\right) \\ \vdots \\ \frac{1}{r_{n}} \frac{\partial \bar{u}_{n}}{\partial x}\left(d_{n}\right)\end{array}\right]+B .}\end{array}\right.$

Solving problem (4.7) is trivial; upon introducing its solution $\bar{u}$ in the boundary condition from (4.8), a problem of the type (4.1), (4.2) (with $k_{0}=0$ ) is obtained. With similar arguments we conclude that, for semi-positive $G$, the operator $A$ is $m$-dissipative in $H_{c}^{0}$. On the other hand, our dynamic problem (2.2), (2.4), (2.5) is equivalent with a Cauchy problem on $H_{c}^{0}$, namely,

$$
\left\{\begin{array}{c}
\frac{d u(t, \cdot)}{d t}=A u(t, \cdot) \\
u(0, \cdot)=u_{0}(\cdot)
\end{array}\right.
$$

The $m$-dissipativity implies that $A$ generates a contraction semigroup of class $C_{0}$, [3], and hence, [23] for any $u_{0} \in$ $\mathscr{D}(A)$ there exists a unique solution of (4.9) belonging to class $C\left([0, \infty), H_{c}^{0}\right) \cap C^{1}([0, \infty), \mathscr{D}(A))$. Finally, from the well-known a priori estimates for parabolic problems combined with Sobolev's imbedding theorem, the desired differentiability properties follow (see [24] and [25] for more classical approach). For recent study of Cauchy problem we refer to [29].

\section{B. Proof of Theorem 3.2}

Let $u=\left(u_{1}, \cdots, u_{n}\right)$ be the classical solution of our problem (2.2), (2.4), (2.5). For every $k \in \overline{1, n}$ we take

$$
u_{k}\left(t, x_{k}\right)=v_{k}\left(t, x_{k}\right) \cos \alpha_{k} \frac{x_{k}}{d_{k}}
$$

where $\alpha_{k}$ will be conveniently chosen. We obtain a new problem

$$
\left\{\begin{aligned}
\frac{\partial v_{k}}{\partial t}= & \frac{1}{r_{k} c_{k}} \frac{\partial^{2} v_{k}}{\partial x^{2}}-\left(\frac{2}{r_{k} c_{k}} \frac{\alpha_{k}}{d_{k}} \operatorname{tg} \frac{\alpha_{k} x_{k}}{d_{k}}\right) \frac{\partial v_{k}}{\partial x} \\
& -\left[\left(\frac{\alpha_{k}}{d_{k}}\right)^{2} \frac{1}{r_{k} c_{k}}+\frac{g_{k}}{c_{k}}\right] v_{k}, \\
& x \in\left[0, d_{k}\right], \quad t \geqslant 0, \quad k \in \overline{1, n}
\end{aligned}\right.
$$


with boundary conditions: (we leave out the argument " $t$ " of $v_{k}$ and $\partial v_{k} / \partial_{x}$ )

$$
\left[\begin{array}{c}
-\frac{1}{r_{1}} \frac{\partial v_{1}}{\partial x}(0) \\
\frac{1}{r_{1}} \frac{\partial v_{1}}{\partial x}\left(d_{1}\right) \cos \alpha_{1} \\
\vdots \\
-\frac{1}{r_{n}} \frac{\partial v_{n}}{\partial x}\left(d_{n}\right) \cos \alpha_{n}
\end{array}\right]=-G\left[\begin{array}{c}
v_{1}(0) \\
v_{1}\left(d_{1}\right) \cos \alpha_{1} \\
\vdots \\
v_{n}(0) \\
v_{n}\left(d_{n}\right) \cos \alpha_{n}
\end{array}\right]+\left[\begin{array}{c}
0 \\
\frac{\alpha_{1} v_{1}\left(d_{1}\right)}{r_{1} d_{1}} \sin \alpha_{1} \\
0 \\
\vdots \\
0 \\
\frac{\alpha_{n} v_{n}\left(d_{n}\right)}{r_{n} d_{n}} \sin \alpha_{n}
\end{array}\right]+B(t)
$$

and with initial conditions

$$
v(0, x)=u_{k 0}(x) / \cos \alpha_{k} \frac{x}{d_{k}} .
$$

The problem (4.11)-(4.13) can be seen as an abstract Cauchy problem on the space

$Z=\left\{f: \prod_{k=1}^{n}\left[0, d_{k}\right] \rightarrow \boldsymbol{R}^{n}\right.$, with components

$$
\left.f_{k}:\left[0, d_{k}\right] \rightarrow \boldsymbol{R} \text { continuous }\right\} \text {. }
$$

For this we put

$\mathscr{D}(\Lambda)=\{f \in Z$, with components twice differentiable

$$
\text { and satisfying (4.12) \} }
$$

and we define $\Lambda: \mathscr{D}(\Lambda) \rightarrow Z$ with components given by the right-hand side of (4.11). Then (4.11)-(4.13) is equivalent with

$$
\left\{\begin{aligned}
\frac{d}{d t} v(t, \cdot) & =\Lambda v(t, \cdot) \\
v(0, \cdot) & =\text { a vector with components given by }(4.13) .
\end{aligned}\right.
$$

The following result is essential for our aims:

Lemma 4.1: We suppose that $G$ is DRD and for every $j \in \overline{1, n}$ we denote $\alpha_{j}=\gamma_{j}-\epsilon$ where $\gamma_{j} \in(0, \pi / 2)$ is the solution of (3.1) and $\epsilon>0$ is such that $\alpha_{j} \in(0, \pi / 2)$. If we take

$$
\omega_{\epsilon}=-\min _{1 \leqslant j \leqslant n}\left[\frac{\left(\gamma_{j}-\epsilon\right)^{2}}{d_{j}^{2} r_{j} c_{j}}+\frac{g_{j}}{c_{j}}\right]
$$

then, the operator $\Lambda-\omega_{\epsilon} I$ constructed with these $\alpha_{j}$ is dissipative on $Z$.

Proof: If we take $v, \bar{v} \in \mathscr{D}(\Lambda)$ and suppose the nontrivial case $w=v-\bar{v} \neq 0$, according with Lemma A (see the Appendix) we have to prove

$$
\sup _{(j ; x) \in M(w)}[\Lambda v-\Lambda \bar{v}]_{j}(x) \operatorname{sgn} w_{j}(x) \leqslant \omega_{\epsilon}\|w\|_{Z} .
$$

Let us take the pair $(p ; y) \in M(w)$, i.e.,

$$
\max _{1 \leqslant i \leqslant n} \max _{1 \leqslant x_{i} \leqslant d_{i}}\left|w_{i}\left(x_{i}\right)\right|=\left|w_{p}(y)\right|=\|w\|_{Z}
$$

and for which clearly we have $w_{p}(y) \neq 0$. If we suppose already proved that $y \neq 0, d_{p}$, two cases may appear. For the first one, i.e., $w_{p}(y)>0, y$ is a maximum point for $w_{p}$ in $\left(0, d_{p}\right)$ and then, from (4.11) we have

$$
\begin{aligned}
{[\Lambda v-} & \Lambda \bar{v}]_{p}(y) \operatorname{sgn} w_{p}(y) \\
= & \frac{1}{r_{p} c_{p}} \frac{\partial^{2} w_{p}}{\partial x^{2}}(y)-\left(\frac{2}{r_{p} c_{p}} \frac{\alpha_{p}}{d_{p}} \operatorname{tg} \frac{\alpha_{p} x_{p}}{d_{p}}\right) \frac{\partial w_{p}}{\partial x}(y) \\
& -\left(\frac{\alpha_{p}^{2}}{d_{p}^{2} r_{p} c_{p}}+\frac{g_{p}}{c_{p}}\right)\left|w_{p}(y)\right| \\
\leqslant & -\left(\frac{\alpha_{p}^{2}}{d_{p}^{2} r_{p} c_{p}}+\frac{g_{p}}{c_{p}}\right)\left|w_{p}(y)\right| \leqslant \omega_{\epsilon}\|w\|_{z} .
\end{aligned}
$$

The same inequality can be obtained in the second case $w_{p}(y)<0$, when $y$ is a minimum point for $w_{p}$. Thus we have shown (4.15). It remains to prove that $y \neq 0, d_{p}$. Let us suppose $y=0$. From odd rows of (4.12), following (4.16) we easily derive

$$
-\frac{1}{r_{p}} \frac{\partial w_{p}}{\partial x}(0) w_{p}(0) \leqslant\left(-G_{2 p-1,2 p-1}+S_{2 p-1}\right)\left|w_{p}(0)\right|^{2}
$$

and from DRD property of $G$ it results

$$
-\frac{1}{r_{p}} \frac{\partial w_{p}}{\partial x}(0) w_{p}(0)<0
$$

But if $w_{p}(0)>0$, then $w_{p}(0) \geqslant w_{i}(x)$ for any $i$ and $x$ and

$$
\left[-\frac{1}{r_{p}} \lim _{x_{p} \rightarrow 0^{+}} \frac{w_{p}\left(x_{p}\right)-w_{p}(0)}{x_{p}}\right] w_{p}(0) \geqslant 0
$$

that contradicts (4.17). Also if $w_{p}(0)<0$, then $w_{p}(0) \leqslant$ $w_{i}(x)$ for any $i$ and $x$ and we obtain again a contradiction. Hence $y \neq 0$.

Let us suppose now $y=d_{p}$. Also, from boundary conditions (4.12) (namely from even rows) and from (4.16) we may derive the following inequality:

$$
\begin{aligned}
& \frac{1}{r_{p}} \frac{\partial w_{p}}{\partial x}\left(d_{p}\right) w_{p}\left(d_{p}\right) \cos \alpha_{p} \\
& \leqslant\left(-G_{2 p, 2 p} \cos \alpha_{p}+S_{2 p}+\frac{\alpha_{p}}{r_{p} d_{p}} \sin \alpha_{p}\right)\left|w_{p}\left(d_{p}\right)\right|^{2}
\end{aligned}
$$

On the other hand, we have $\cos \alpha_{p}>\cos \gamma_{p}=$ the right- 
hand side member of (3.1) (with $j=p$ ) and this implies

$$
-\left(G_{2 p, 2 p}+\frac{1}{r_{p} d_{p}}\right) \cos ^{2} \alpha_{p}+S_{2 p} \cos \alpha_{p}+\frac{1}{r_{p} d_{p}}<0 .
$$

From here, with the inequality $\alpha_{p}<\tan \alpha_{p}$ we derive that

$$
-G_{2 p, 2 p} \cos \alpha_{p}+S_{2 p}+\frac{\alpha_{p}}{r_{p} d_{p}} \sin \alpha_{p}<0
$$

and (4.18) gives us

$$
\frac{1}{r_{p}} \frac{\partial w_{p}}{\partial x}\left(d_{p}\right) w_{p}\left(d_{p}\right) \cos \alpha_{p}<0
$$

But, as above, is easy to see that we have obtained a contradiction with $\left(p ; d_{p}\right) \in M(w)$. Then $y \neq d_{p}$ and the proof of Lemma 4.1 is finished.

Now we are in position to obtain the result of Theorem 3.2. If we denote by $v$ and $\bar{v}$ the correspondent functions of $u$ and $\bar{u}$, respectively, via (4.10) with $\alpha_{k}=\gamma_{k}-\epsilon$ and $\gamma_{k}$ given by (3.1), these functions satisfy (4.14) resulting in

$$
\frac{d}{d t}[v(t, \cdot)-\bar{v}(t, \cdot)]=\Lambda v(t, \cdot)-\Lambda \bar{v}(t, \cdot) .
$$

On the other hand, the norm $\|v(t, \cdot)-\bar{v}(t, \cdot)\|_{Z}$ is differentiable on $\mathscr{M}=[0, \infty)-\mathscr{N}$ where $\mathscr{N}$ is an at most countable set. A known result of Coppel [27] combined with (4.18) gives us

$$
\begin{aligned}
\frac{d}{d t} \| v(t, \cdot)-\bar{v}(t, \cdot) & \|_{Z} \\
& =\sigma(v(t, \cdot)-\bar{v}(t, \cdot), \Lambda v(t, \cdot)-\Lambda \bar{v}(t, \cdot))
\end{aligned}
$$

for any $t \in \mathscr{M}$. From here and from the previous lemma we obtain on $\mathscr{M}$,

$$
\frac{d}{d t}\|v(t, \cdot)-\bar{v}(t, \cdot)\|_{Z} \leqslant \omega_{\epsilon}\|v(t, \cdot)-\bar{v}(t, \cdot)\|_{Z}
$$

Hence $\|v(t, \cdot)-\bar{v}(t, \cdot)\|_{Z} \leqslant\|v(0, \cdot)-\bar{v}(0, \cdot)\|_{Z} e^{\omega_{c} t}$ for every $t \geqslant 0$. From this relation, with (4.10) and $\epsilon \rightarrow 0$, we easily derive the inequality from theorem. Also, because $\omega_{\epsilon}<0$, (4.20) gives the desired monotony.

\section{Approximation of the SOlution}

We shall approximate the solution $u$ of the problem (2.2), (2.4), (2.5) by the finite difference method in time and the finite element method in space. If $\phi=\left(\phi_{1}, \cdots, \phi_{n}\right)$ $\in H^{1}=\prod_{k=1}^{n} H_{c_{k}}^{1}\left(0, d_{k}\right)$, then by (4.9),

$$
\left\{\begin{aligned}
\left\langle\frac{d}{d t} u(t), \phi\right\rangle_{H^{0}} & =\langle A u, \phi\rangle_{H^{0}} \\
\langle u(0), \phi\rangle_{H^{0}} & =\left\langle u_{0}, \phi\right\rangle_{H^{0}}
\end{aligned}\right.
$$

where $\langle\cdot, \cdot\rangle_{H^{0}}$ denotes the inner product in $H^{0}=$ $\prod_{k=1}^{n} L_{c_{k}}^{2}\left(0, d_{k}\right)$.

If we formally integrate by parts, then according with (2.5) we get that

$$
\frac{d}{d t}\langle u(t), \phi\rangle_{H^{0}}+P(u(t), \phi)=R(B(t), \phi)
$$

for all $\phi \in H^{1}$, where

$P(u(t), \phi)$

$$
\begin{aligned}
= & \sum_{k=1}^{n} \frac{1}{c_{k}}\left\{\int _ { 0 } ^ { d _ { k } } \left[\frac{1}{r_{k}} \frac{\partial u_{k}}{\partial x}(t, x) \frac{\partial \phi_{k}}{\partial x}(x)\right.\right. \\
& \left.+g_{k} u_{k}(t, x) \phi_{k}(x)\right] d x \\
& +\phi_{k}(0) \cdot \sum_{j=1}^{n}\left[G_{2 k-1,2 j} u_{j}(0)+G_{2 k-1,2 j-1} u_{j}\left(d_{j}\right)\right] \\
& \left.+\phi_{k}\left(d_{k}\right) \cdot \sum_{j=1}^{n}\left[G_{2 k, 2 j} u_{j}(0)+G_{2 k-1,2 j-1} u_{j}\left(d_{j}\right)\right]\right\}
\end{aligned}
$$

and

$$
R(B(t), \phi)=\sum_{k=1}^{n} \frac{1}{c_{k}}\left[b_{2 k-1}(t) \phi_{k}(0)+b_{2 k}(t) \phi_{k}\left(d_{k}\right)\right] .
$$

To approximate (5.2) we suppose that a family of finite dimensional subspaces $\left\{S_{h}\right\}_{h>0}$ of $H^{1}$ is given such that for a small positive $h$ there is a constant $c$, independent of $h$ and $v$ for which

$$
\inf _{\phi \in S_{h}}\left\{\|v-\phi\|_{H^{0}}+h\|v-\phi\|_{H^{1}}\right\} \leqslant c h^{s}\|v\|_{H^{s}}
$$

where $v \in H^{s}$ for $s=2$. For examples of such subspaces, which include the usual piecewise-linear finite element spaces see [28].

We may now pose the semi-discrete problem corresponding to (5.2). Find $u_{h}(t) \in S_{h}$ for $t>0$, such that

$$
\left\{\begin{array}{l}
\frac{d}{d t}\left\langle u_{h}(t), \phi\right\rangle_{H^{0}}+P\left(u_{h}(t), \phi\right)=R(B(t), \phi) \\
\left\langle u_{h}(0), \phi\right\rangle_{H^{0}}=\left\langle u_{0}, \phi\right\rangle_{H^{0}}, \\
\text { for all } \phi \in S_{h} .
\end{array}\right.
$$

Once a basis for the finite-dimensional space

$$
S_{h}=S_{h}^{1} \times \cdots \times S_{h}^{n}, \quad\left\{\phi_{1}^{1}, \cdots, \phi_{N_{1}}^{1}, \cdots, \phi_{1}^{n}, \cdots, \phi_{N_{n}}^{n}\right\}
$$

is given $\left(N_{i}=N_{i}(h)\right.$ is the dimension of $\left.S_{h}^{i}\right)$, we can write

$$
u_{h}(t)=\sum_{i=1}^{n} \sum_{j=1}^{N_{i}} Q_{j}^{i}(t) \phi_{j}^{i} \text {. }
$$

In this way, the problem (5.3) comes to a system of ordinary differential equations of the form:

$$
\left\{\begin{array}{r}
M Q^{\prime}(t)+K Q(t)=F(t) \\
Q(0)=Q_{0}
\end{array}\right.
$$

where the mass and the stiffness matrices are, respectively,

$$
M=\left\{\left\langle\phi_{i}^{j}, \phi_{k}^{l}\right\rangle_{H^{0}}\right\} \text { and } K=\left\{P\left(\phi_{i}^{j}, \phi_{k}^{l}\right)\right\} .
$$

The system (5.4) can be solved numerically, for example, by implicit Euler difference scheme. If $\Delta t$ denotes the time mesh size, and $Q^{k}=Q(k \Delta t), F^{k}=F(k \Delta t)$, then

$$
\left\{\begin{aligned}
\left(\frac{M}{\Delta t}+K\right) Q^{k+1} & =F^{k+1}+\frac{M}{\Delta t} Q^{k}, \quad k=0,1, \cdots \\
Q^{0} & =Q_{0}
\end{aligned}\right.
$$




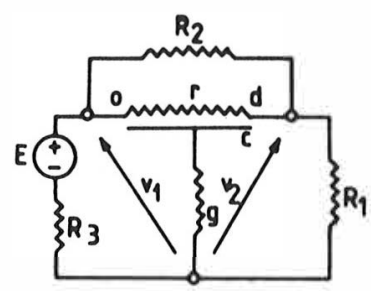

Fig. 3. The network for Examples I, II, and III.

TABLE I

\begin{tabular}{|ccccc|}
\hline Ex. & $r\left(\Omega \mathrm{m}^{-1}\right)$ & $c\left(\mathrm{Fm}^{-1}\right)$ & $g\left(\Omega^{-1} \mathrm{~m}^{-1}\right)$ & $d(\mathrm{~m})$ \\
6.1 & 4000 & 9000 & 0.001 & 0.001 \\
6.2 & 4000 & 9 & 0.001 & 0.001 \\
6.3 & 400 & 0.9 & 0.0001 & 0.01 \\
\hline
\end{tabular}

\section{NUMERICAL EXAMPLES}

Let us consider a very simple network (Fig. 3 ) with the resistive part having the values $R_{1}=5 \Omega, R_{2}=5 \Omega, R_{3}=$ $1 \Omega, E=1 \mathrm{~V}$ and with the distributed parameters from Table I (three variants, Examples I-III).

The matrix $G$ and the vector $B$ have the following terms: $G_{11}=R_{2}^{-1}+R_{3}^{-1}=1.2, G_{22}=R_{1}^{-1}+R_{2}^{-1}=0.4, G_{12}$ $=G_{21}=-R_{2}^{-1}=-0.2, b_{1}=E / R_{3}=1, b_{2}=0$. The matrix $G$ is symmetric and DRD. This is why our network has unique dynamic and steady-state solutions and by (3.1), (3.2), (3.4), (3.6) we can calculate $\bar{D}(t), \bar{T}_{0.1}, \bar{T}_{0.5}$.

On the other hand, we have computed numerically our problem by the method presented in Section V. In Fig. 4 we give the space variation of the solution for the different values $t_{i}=i \Delta t$ of the time in the case of Example I.

The discretization parameters were $h=0.0202 \cdot d$ and $\Delta t=8.52$. For the same example, in Fig. 5 the numerically comptued $D(t), T_{0.1}, T_{0.5}$ together with their a priori upper bounds are given.

The results for the delay time in Examples I-III are summarized in Table II.

As expected by intuition, the delay time $T_{\lambda}$ is (approximately) the same in Examples II and III, where the global parameters $r d, c d$, and $g d$ have been chosen to be the same. A high mark of our bound $\bar{T}_{\lambda}$ is the fact that it is also the same for the two cases.

Let us consider now the simplest amplifier with one bipolar transistor and with two distributed elements (Fig. 6).

In Fig. 7 the same circuit is plotted with the incremental equivalent model for the transistor.

The physical parameters are:

- for the resistances: $R_{1}=50 \Omega, R_{2}=$ $10^{3} \Omega, R_{C}=10^{3} \Omega, R_{e}=12235 \Omega$.

- for the small signal model: $\beta=50, h=10^{3} \Omega$.

- for the distributed elements: $r_{1}=2 \times 10^{7} \Omega \cdot \mathrm{m}^{-1}, c_{1}=$ $2 \times 10^{-8} \mathrm{~F} \cdot \mathrm{m}^{-1}, g_{1}=0, d_{1}=5 \times 10^{-3} \mathrm{~m}, r_{2}=5 \times$ $10^{5} \Omega \cdot \mathrm{m}^{-1}, \quad c_{2}=5 \times 10^{-6} \mathrm{~F} \cdot \mathrm{m}^{-1}, g_{2}=0, d_{2}=2 \times$ $10^{-3} \mathrm{~m}$.

With these parameters $G$ is a nonsymmetric but positive definite and DRD matrix: $G_{11}=2 \times 10^{-2} \Omega^{-1}, G_{22}=1.6 \times$



Fig. 4. Numerically computed solutions in Example I.

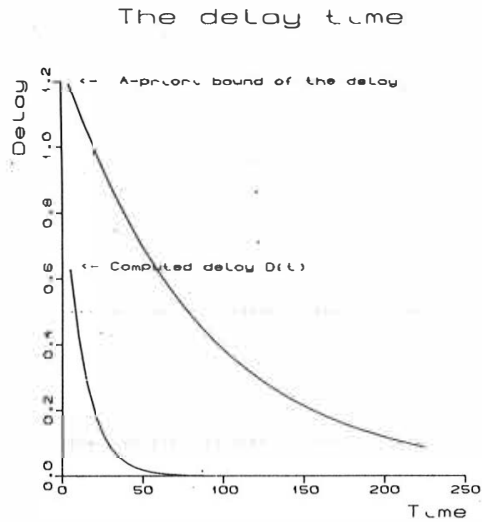

Fig. 5. $D(t)$ and $\bar{D}(t)$ in Example I.

TABLE II

\begin{tabular}{|ccccccc|}
\hline Ex. & $\omega_{0}\left(\mathrm{~s}^{-1}\right)$ & $\cos \gamma$ & $T_{0.1}(\mathrm{~s})$ & $\bar{T}_{0.1}(\mathrm{~s})$ & $T_{0.5}(\mathrm{~s})$ & $\bar{T}_{0.5}(\mathrm{~s})$ \\
6.1 & -0.0119 & 0.7928 & $\sim 43$ & 213 & $\sim 17$ & 77.758 \\
6.2 & -11.9429 & 0.7928 & $\sim 0.043$ & 0.213 & $\sim 0.017$ & 0.077 \\
6.3 & -11.9429 & 0.7928 & $\sim 0.043$ & 0.213 & $\sim 0.017$ & 0.077 \\
\hline
\end{tabular}

$10^{-6} \Omega^{-1}, G_{33}=10^{-3} \Omega^{-1}, G_{44}=10^{-3} \Omega^{-1}, G_{32}=8 \times 10^{-5}$ $\Omega^{-1}$ and other components are $=0$. For $B$, only the first element is nonzero: $b_{1}=2 \times 10^{-2} \mathrm{~A}$.

After a calculus with formulas from Section III we obtain

$$
\omega_{0}=-14490 \mathrm{~s}^{-1} \text { and } \min \left\{\cos \gamma_{1}, \cos \gamma_{2}\right\}=0.707 .
$$

These give a priori bounds of response time:

$$
\bar{T}_{0.1}=182.8 \mu \mathrm{s} \text { and } \bar{T}_{0.5}=71.76 \mu \mathrm{s} \text {. }
$$

On the other hand, the application of the numerical method of Section $\mathrm{V}$ provides the results shown in Fig. 8 for $u_{1, h}(t, x)$ and in Fig. 9 for $u_{2, h}(t, x)$. The discretization parameters are $h_{1}=0.0202 \cdot d_{1}, h_{2}=0.0202 \cdot d_{2}, \Delta t=1.8 \times$ $10^{-6}$. Numerical calculus gives $T_{0.1}=14.4 \mu \mathrm{s}$ and $T_{0.5}=$ $7.2 \mu \mathrm{s}$.

In Fig. 10 we can see the computed $D(t)$ and its a priori bound.

All above examples show that our bounds are sufficiently tight for a first evaluation, if we take into account how simple they are to calculate. Certainly, a similar lower bound of the delay time is desirable. 


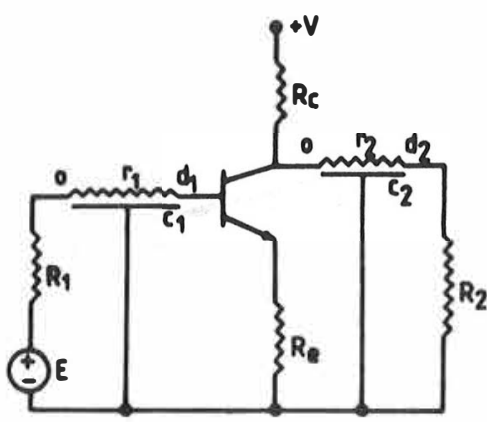

Fig. 6. A simple transistor network.

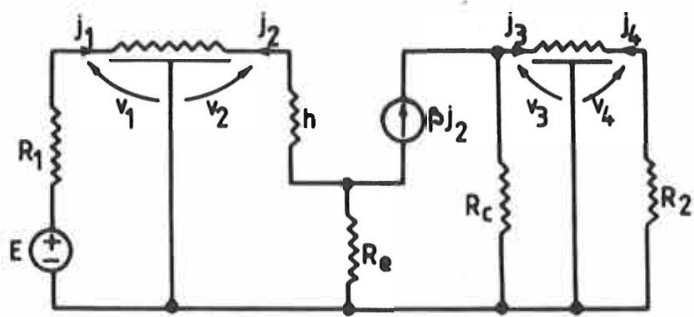

Fig. 7. The equivalent circuit.

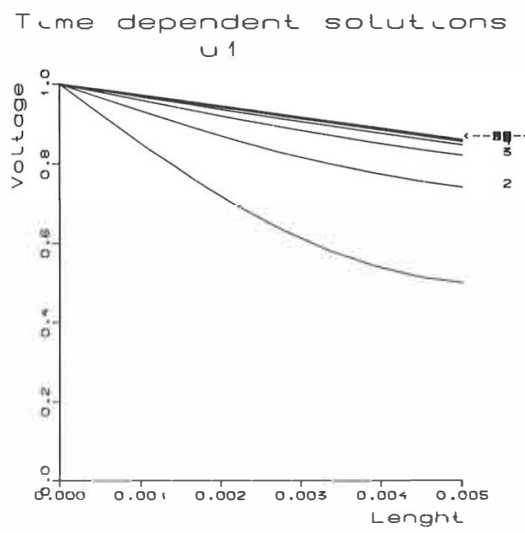

Fig. 8. Numerically computed $u_{1}(t, x)$.

\section{CONClusions}

The complexity of circuits models whose consistency is assured by our theorems is limited by the existence of the description matrix of the resistive multiport and by its properties needed as hypotheses. As we saw, the area of applicability of the results is large enough.

As regards the delay time, in many cases this quantity is useful as a comparison criterium. For instance, in circuit design, in view to obtain from a circuit $C_{1}$ "a two times faster" circuit $C_{2}$, we must change the parameters such that $T_{\lambda}^{2} / T_{\lambda}^{1}=2$. If the equality $\bar{T}_{\lambda}^{2} / \bar{T}_{\lambda}^{1}=T_{\lambda}^{2} / T_{\lambda}^{1}$ is (at least approximately) satisfied, then $\bar{T}_{\lambda}$ itself can be considered as "the delay time" of the network. Examples I, II, III (regarding the same circuit) show that this is really the case.

\section{APPENDIX}

If $X$ is a real Banach space with the norm \|\|$_{X}$, let us consider the functional $\sigma: X \times X \rightarrow \boldsymbol{R}$ with $\sigma(a, b)=$ $\lim _{h \rightarrow 0+}\left(\|a+h b\|_{X}-\|a\|_{X}\right) / h$. We shall say that $f: \mathscr{D} \subset$

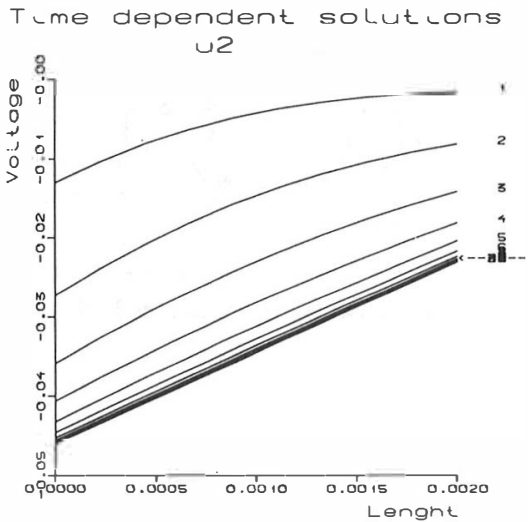

Fig. 9. Numerically computed $u_{2}(t, x)$.

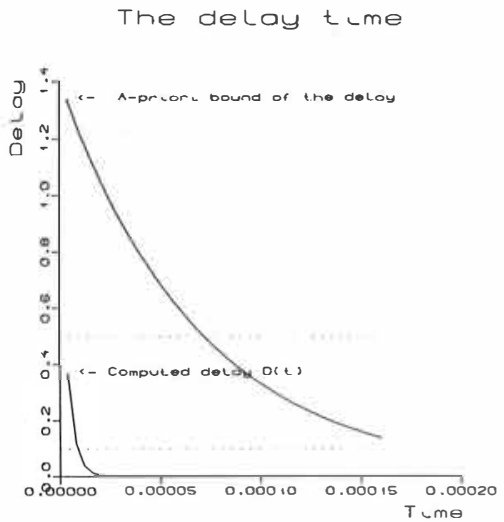

Fig. 10. $D(t)$ and $\bar{D}(t)$.

$X \rightarrow X$ is dissipative if $\sigma(x-y, f(x)-f(y)) \leqslant 0$ for any $x, y \in \mathscr{D}$. If in addition Range $(I-f)=X$, then $f$ is said to be $m$-dissipative. If $\alpha$ is a real number, it is easily to show that $f-\alpha I$ is dissipative iff for every $x, y \in \mathscr{D}$,

$$
\sigma(x-y, f(x)-f(y)) \leqslant \alpha\|x-y\|_{X} .
$$

For a Hilbert space $X$ with the inner product $\langle\cdot, \cdot\rangle_{X}$, we find $\|a\|_{X} \sigma(a, b)=\langle a, b\rangle_{X}$, so that $f$ is dissipative iff $\langle x-y, f(x)-f(y)\rangle_{X} \leqslant 0$ for any $x, y \in \mathscr{D}$.

Finally, if $Y$ is a compact space, let us consider the Banach space $C=C\left(Y, \boldsymbol{R}^{n}\right)=\left\{f: X \rightarrow \boldsymbol{R}^{n}\right.$ with continuous $f_{i}$ components $\}$ with the usual supremum norm. In order "to calculate" the $\sigma$ functional in this space, we shall give a result which is an extension of a theorem of Sato [26], with a simpler proof.

Lemma $A$ : For $f, g \in C, f \neq 0$, it holds

$$
\sigma(f, g)=\sup _{(p ; x) \in M(f)} g_{p}(x) \operatorname{sgn} f_{p}(x)
$$

where

$$
M(f)=\left\{(p ; \bar{x})\left|\max _{1 \leqslant i \leqslant n} \max _{x \in Y}\right| f_{i}(x)|=| f_{p}(\bar{x}) \mid=\|f\|_{C}\right\} .
$$

Proof: Let $\left\{\epsilon_{k}\right\}_{k=1}^{\infty}$ with $\epsilon_{k} \rightarrow 0^{+}$for $k \rightarrow \infty$. For every pair $\{k, i\}$ of indexes we choose $x_{k, i} \in Y$ such that

$$
\max _{x \in Y}\left|f_{i}(x)+\epsilon_{k} g_{i}(x)\right|=\left|f_{i}\left(x_{k, i}\right)+\epsilon_{k} g_{i}\left(x_{k, i}\right)\right| .
$$


Consequently,

$$
\begin{aligned}
\left\|f+\epsilon_{k} g\right\|_{C} & =\max _{i}\left|f_{i}\left(x_{k, i}\right)+\epsilon_{k} g_{i}\left(x_{k, i}\right)\right| \\
& =\left|f_{p}\left(x_{k, p}\right)+\epsilon_{k} g_{p}\left(x_{k, p}\right)\right| .
\end{aligned}
$$

We choose from $\left\{x_{k, p}\right\}_{k=1}^{\infty}$ a convergent subsequence (keeping the same notation) $\left\{x_{k, p}\right\}_{k=1}^{\infty} \rightarrow x_{p}$, for $k \rightarrow \infty$. By (A.1), $\left(p, x_{p}\right) \in M(f)$. On the other hand, it is easy to see that there exists an index $N$ such that for $k>N$ we have $\operatorname{sgn}\left[f_{p}\left(x_{k, p}\right)+\epsilon_{k} g_{p}\left(x_{k, p}\right)\right]=\operatorname{sgn} f_{p}\left(x_{k, p}\right)=$ sgn $f_{p}\left(x_{p}\right)$. Hence, taking also into account the relation (A.1) we obtain for $k>N$ :

$$
\begin{aligned}
& \frac{\left\|f+\epsilon_{k} g\right\|_{C}-\|f\|_{C}}{\epsilon_{k}} \\
& \quad \leqslant \frac{\left|f_{p}\left(x_{k, p}\right)+\epsilon_{k} g_{p}\left(x_{k, p}\right)\right|-\left|f_{p}\left(x_{k, p}\right)\right|}{\epsilon_{k}} \\
& \quad=g_{p}\left(x_{k, p}\right) \operatorname{sgn} f_{p}\left(x_{p}\right) .
\end{aligned}
$$

From here we find

$$
\sigma(f, g) \leqslant g_{p}\left(x_{p}\right) \operatorname{sgn} f_{p}\left(x_{p}\right) \leqslant \sup _{(p ; x) \in M(f)} g_{p}(x) \operatorname{sgn} f_{p}(x) .
$$

In order to give the converse part of the proof, we take $\left(p, x_{p}\right) \in M(f)$ and we observe that

$$
\frac{\|f+\epsilon g\|_{C}-\|f\|_{C}}{\epsilon} \geqslant\left|f_{p}\left(x_{p}\right)\right| \frac{\left|1+\frac{g_{p}\left(x_{p}\right)}{f_{p}\left(x_{p}\right)}\right|-1}{\epsilon} .
$$

Due to the fact that the right-hand side tends to $\left|f_{p}\left(x_{p}\right)\right|\left(g_{p}\left(x_{p}\right) / f_{p}\left(x_{p}\right)\right)$ when $\epsilon$ tends to $0^{+}$, the inequality follows.

\section{ACKNOWLEDGMENT}

The authors would like to express their gratitude to the reviewers for their valuable comments and suggestions. Also we are indebted to P. Koikkalainen for his assistance in numerical tests.

\section{REFERENCES}

[1] K. C. Saraswat, and F., Mohammedi, "Effect of scaling of interconnections on the time delay of VLSI circuits," IEEE Trans. Electr. Dev., vol. ED-29, Apr. 1982.

[2] J. Rubinstein, P. Penfield, and M. Horowitz, "Signal delay in $R C$ tree networks," IEEE Trans. Comp.-Aided Design, vol. CAD-2, pp. 202-211, July 1983.

[3] H. Brézis, "Operateurs Maximaux Monotones et Semigroupes de Contractions daus les Espáces de Hilbert," North-Holland Publ. Comp., 1973.

[4] R. H. Martin, Jr., Nonlinear Operators and Differential Equations in Banach Spaces. New York: Wiley, 1976.

[5] V. Barbu, "Nonlinear boundary value problems for a class of hyperbolic systems," Rev. Roum. Math. Pures Appl., vol. 22, pp. $155-168,1977$

[6] G. Morosanu, "Existence for nonlinear differential systems of hyperbolic type," An. Stiint, Univ. Al. I. Cuza, Iasi, Sect I Mat., vol. 26, pp. 345-352, 1980.

[7] "Stability of solutions of nonlinear boundary value problems for hyperbolic systems," Nonlinear Analysis, TMA 5, pp. 61-70, 1981.

[8] Mixed problems for a class of nonlinear differential hyperbolic systems, J. Math. Anal. Appl., vol. 83, pp. 470-485, 1981.

[9] G. Prada and T. A. Bickart, "Stability of electrical networks containing distributed RC components," J. Math. Anal. Appl., vol. 33 , pp. $367-401,1971$.
[10] R. K. Brayton, "Small signal stability criterion for electrical networks containing lossless transmission lines," IBMJ. Res. Develop., vol. 12, pp. 431-440, 1968.

[11] C. A. Desoer, "Distributed networks with small parasitic elements: input-output stability," IEEE Trans. Circuits Syst., vol. CAS-24, pp. 1-7, 1977.

[12] W. C. Elmore, "The transient response of damped linear networks with particular regard to wide-band amplifiers," J. Appl. Phys., vol. 19, pp. 55-63, 1948.

[13] E. N. Protonotarios and O. Wing, "Theory of nonuniform $R C$ lines, Part II: Analytic properties in the time domain," IEEE Trans. Circuit Theory, vol. 14, pp. 13-20, 1967.

[14] K. Singhal and J. Vlach, "Approximation of nonuniform $R C$-distributed networks for frequency and time-domain computations," IEEE Trans. Circuit Theory, vol. CT-19, pp. 347-354, 1972.

[15] T. Sakurai, "Approximation of wiring delay in MOSFET LSI," IEEE J. Solid-State Circuits, vol. SC-18, pp. 418-426, Aug. 1983.

[16] T. Tokuda, K. Okazaki, K. Sakashita, I. Ohkura, and T. Enomoto, "Delay time modeling for ED MOS logic LSI," IEEE Trans. Comp--Aided Design, vol. CAD-2, pp. 129-134, 1983.

[17] T. M. Lin, and C. A. Mead, "Signal Delay in general $R C$ networks," IEEE Trans. Comp.-Aided Design, vol. CAD-3, pp. 331-349, Oct. 1984.

[18] J. L. Wyatt, Jr. and Yu Qingjian, "Signal delay in $R C$ meshes, trees and lines," in Proc. IEEE Int. Conf. on CAD (ICCAD 84), Santa Clara, CA, pp. 15-17, Nov. 1984.

[19] J. L. Jr., Wyatt, "Monotone sensitivity of nonlinear nonuniform $R C$ transmission lines, with application to timing analysis of digital MOS integrated circuits," IEEE Trans. Circuits Syst., vol. CAS-32, pp. 28-33, Jan. 1985.

[20] C. Marinov, "Qualitative properties of $I^{P}$-solutions of infinite differential systems via dissipativity," Nonlinear Analysis TMA 8 pp. $441-456,1984$

[21] C. Marinov and P. Neittaanmäki, The problem of response time in electrical circuits with resistively coupled distributed structures, Research Report 8, Lappeenranta Univ. of Techn., Finland (1985).

[22] I. W. Sandberg and A. N. Willson, Jr. "Some theorems on properties of d.c. equations of nonlinear networks," Bell Syst. Tech. J., vol 48 No 1 (Jan. 1969), 1-34, Section IX.

[23] J. A. Goldstein, "Semigroups of operators and abstract Cauchy problems," Tulane Univ. Lecture Notes, 1970.

[24] J. L. Lions, Non-Homogeneous Boundary Value Problems and Applications II. Berlin, Germany: Springer Verlag, 1972

[25] G. Hellwig, Differential Operators of Mathematical Physics. Reading, MA: Addison-Wesley, 1967.

[26] K. Sato, "On the generators of non negative contraction semi-groups in Banach lattices," J. Math. Soc. Japan, vol. 20, pp. 423-436, 1968.

[27] W. A. Coppel, Stability and Asymptotic Behavior of Differential Equations. Boston, MÁ: Health, 1965.

[28] G. Strang and G. J. Fix, Analysis of the Finite Element Method. Englewood Cliffs, NJ: Prentice-Hall, 1973.

[29] H. O. Fattorini, "The Cauchy Problem," Encyclopedia of mathematics and its applications, vol. 18, Addison-Wesley, Reading, 1983.

[30] C. A. Marinov, "The delay time for a rcg line," Int. J. Circuit Th. Appl., vol. 15, pp. 79-83, 1987. 